\title{
Article \\ Diagnostic relations between pressure and entropy perturbations for acoustic and entropy modes
}

\author{
Sergey Leble ${ }^{1, \ddagger *}$ and Ekaterina Smirnova ${ }^{1, \ddagger}$ \\ 1 Immanuel Kant Baltic Federal University, Kaliningrad, Kaliningrad Oblast 236041, Russia; \\ * Correspondence: lebleu@mail.ru \\ $\ddagger$ These authors contributed equally to this work.
}

\begin{abstract}
Diagnostics and decomposition of atmospheric disturbances in a planar flow are considered and applied to numerical modeling results with the direct possibility to use in atmosphere monitoring especially in such strong events which follow magnetic storms. The study examines a situation in which the stationary equilibrium temperature of a gas may depend on a vertical coordinate, that seriously complicates the problem solution. The relations connecting perturbations for acoustic and entropy modes are analytically established and led to the solvable diagnostic equations. These perturbation structures, found as the equation solutions specify acoustic and entropy modes in an arbitrary stratified gas under the condition of stability. These time-independent diagnostic relations link gas perturbation variables of the acoustic and the entropy modes. Hence, they provide the ability to decompose the total vector of perturbations into acoustic and non-acoustic (entropy) parts uniquely at any instant within the all accessible heights range. As a prospective model, we consider the diagnostics at the height interval $[120 ; 180] \mathrm{km}$, where the equilibrium temperature of a gas depends linearly on the vertical coordinate. For such a heights range it is possible to proceed with analytical expressions for pressure and entropy perturbations of gas variables. Individual profiles of acoustic and entropy parts for some data, obtained by numerical experiment, are illustrated by the plots for the pure numerical data against ones obtained by the model. The total energy of a flow is determined for both approaches and its height profiles are compared.
\end{abstract}

Keywords: acoustics of non-uniform media, wave mode diagnostics,entropy mode, initialization of hydrodynamic field

\section{Introduction}

Theoretical and numerical models, that describe dynamics of gases and liquids affected by external forces are of great interest in geophysics, meteorology, and wave theory [15]. The external forces and sources of energy, as well as momentum transfer, make the background of a fluid non-uniform. Hence, equilibrium thermodynamic parameters should depend on spatial coordinates. It drastically complicates the definition of linear modes (motions of infinitely small magnitude) taking place in such non-uniform media, so-called "non-exponential". The number of roots of the dispersion equation, if it is possible to determine them, agrees with the number of types of motion, and equals the number of balance equations [2]. Each of the balance equations represents a PDE which contains the first-order derivative with respect to time. In the case of isothermal gas in equilibrium with pressure and density depending exponentially on the coordinate (named often the "exponential atmosphere"), and in the simplest case of a planar flow, the dispersion relations may be introduced over the total wavelength range. Such a model is widely used for the classification of wave modes in practice as a "zero approximation". The realistic nonexponential case needs either consideration of the atmosphere as a layered medium or, for the short waves, making use of the Wentzel, Kramers, Brillouin (WKB) method [1].

There are three types of motion in a one-dimensional (1D) exponential atmosphere: two acoustic modes of different direction of propagation, and the entropy mode, corresponding to zero frequency for a linear lossless flow. The entropy mode, however, is not stationary in a viscous fluid that conducts heat, and with a non-linearity account $[2,10]$. In the flows exceeding one dimension, the buoyancy, or "internal" waves appear [1,2]. For 
Rossby and Poincare waves description see, e.g. [2,5] The first results that allow distinguishing modes due to relations of specific perturbations have been obtained namely relative to the motion of an exponentially stratified ideal gas in the constant gravitational field $[5,8,10]$. Mathematically, such relations are fixed as ones of eigenvectors and corresponding projecting operators of the evolution operator, defined by the basic balance system.

Experimental observation of wave and non-wave disturbances is not easy, there are special facilities as "Sura" [15] based on the active experiment of ionosphere excitation that allows measuring directly the atmosphere parameters variations at the ionosphere heights [16]. Only recently an attempt to apply the diagnostic method, based on projecting operators technique, was realized within a set of such measurements. The projecting technique was developed for a space-evolution operator, that allows to apply it to atmosphere parameters relations at a vicinity of a point of observation. It allowed to distinguish upand down- directed acoustic wave via the real dataset [17], in this work the algorithm of entropy mode diagnosis was elaborated, see also [18].

This work considers the dynamics of ideal atmosphere gas perturbations over a background of equilibrium temperature, dependent on height, affected by a constant mass force and other geophysical impacts. The main aim of this study is the diagnostics as decomposition of a disturbance to wave and non-wave modes in the case of arbitrary stable stratification. This is helpful in the interpretation of experimental data related to the significantly disturbed atmosphere (e.g. by storms), it also may be useful in a validation of a numerical modelling [6]. Especially, it is important in establishing the location of wave sources, and modelling the atmosphere's warming $[7,13]$, related to the atmosphere gas wave heating. The theory should base on the balance equations and rely upon physically justified boundary conditions and simplifications [23], its mode decomposition should be instructive in a specific mathematical statement of problem formulation [24,25].

In this study, which develops ideas of [11], the modes of a planar flow are determined by means of relations between specific perturbations that are time-independent. We name such relations as "diagnostic equations". They are valid for arbitrary dependence of the equilibrium temperature on a coordinate for the case of a stable atmosphere. These relations give the ability to distinguish modes from the total field analytically at any instant, to predict their dynamics, and to conclude about the energy of any of them (which remains constant in time). This is undoubtedly important in applications in meteorology and diagnostics of atmospheric dynamics, including the understanding of such phenomena as variations of the equilibrium temperature of the stratosphere, e.g. so-called warming [12] conventionally understood as period-average. Such phenomenon may be explained in the framework of non-linear interaction of sound and entropy modes in presence of a dissipation [7,19], named as "heating" in laboratory acoustics. The whole exposition is also important in the diagnostics of wave and non-wave modes in order to follow experimental observations and numerical simulations [6] as an element of atmosphere dynamics monitoring.

As the practical example of the general theory and the particular model applications we use the dataset of numerical modelling of an atmospheric perturbation by the source, positioned at the vicinity of Earth surface [23],[24]. The theory uses the standard atmosphere $H(z)$ profile [22] at the $z \in[0 ; 500] \mathrm{km}$ range with the application of diagnostic equations solution with the RHS, discretized as the dataset from a numerical experiment we use. We, however, should omit intervals of instability with non-positive energy density. To proceed we choose a diagnostic at the interval at which the $H(z)$ profile is well approximated by a linear function. It is the heights range $z \in[120 ; 180] \mathrm{km}$, for which we elaborate the model with the explicit form of the diagnostic equation solution. For such interval, we compare the results of the general theory digitization and the result of more compact model, based on explicit approximation of the $H(z)$ profile at the pointed height range.

We begin from the basic system of balance equations and derive the diagnostic ones (Sec. 2). In the final subsection, we solve the differential diagnostic equation by the method of factorization. Next, we apply the obtained relation to the datasets, obtained by numerical 
solution of an atmosphere perturbation problem [23] within the heights range $z \in[120 ; 180]$ $\mathrm{km}$, using the $H(z)$ profile from standard atmosphere [22]. It results in entropy mode contributions profiles (Sec. 3). In the Sec. 4 we build the model for the mentioned heights interval repeating the calculations, when possible, analytically, see also [9]. The results, obtained by the direct applications of the theory to the dataset on the base of the standard atmosphere profile within the range of approximate linearity and the model results are compared.

\section{Diagnostic relations}

\subsection{Basic balance equations for arbitrary stable stratification}

The case of the non-exponential atmosphere in equilibrium permits to fix the entropy and acoustic mode without subdivision into "upwards" and "downwards" directed acoustic waves [11], see also [8]. The main functional parameter in this case, the local atmosphere's scale height $H(z)$ depends on height as, e.g. in [22]. The background density which supports the equilibrium distribution of temperature $\bar{T}(z)$, takes the form

$$
\bar{\rho}(z)=\frac{\bar{\rho}(0) H(0)}{H(z)} \exp \left(-\int_{0}^{z} \frac{d z^{\prime}}{H\left(z^{\prime}\right)}\right),
$$

where the pressure scale height is

$$
H(z)=\frac{\bar{p}}{\bar{\rho} g}=\frac{\bar{T}(z)\left(C_{p}-C_{v}\right)}{g} .
$$

It is convenient to introduce the quantity $\varphi^{\prime}$ instead of perturbation in density,

$$
\varphi^{\prime}=p^{\prime}-\gamma \frac{\bar{p}}{\bar{\rho}} \rho^{\prime}
$$

We will name it the entropy perturbation, because in a limit with $g=0$, constant background temperature $\bar{T}, \varphi^{\prime}$ represents the deviation of the thermodynamic process occurring in fluid from adiabatic one.

As it was done in [9] we use the conventional set of variables:

$$
\begin{gathered}
P=p^{\prime} \cdot \exp \left(\int_{0}^{z} \frac{d z^{\prime}}{2 H\left(z^{\prime}\right)}\right), \\
\Phi=\varphi^{\prime} \cdot \exp \left(\int_{0}^{z} \frac{d z^{\prime}}{2 H\left(z^{\prime}\right)}\right), \\
U=V \cdot \exp \left(-\int_{0}^{z} \frac{d z^{\prime}}{2 H\left(z^{\prime}\right)}\right),
\end{gathered}
$$

where $P, \Phi, U$ are the new quantities defined in this way (Eqs, $(4,5,6))$ and $V$ is the vertical velocity of the flow. The system of momentum-energy-mass balance equations in new variables reads (see $[8,9])$ :

$$
\begin{gathered}
\frac{\partial U}{\partial t}=\frac{1}{\bar{\rho}(0)}\left(\frac{\gamma-2}{2 \gamma H(0)}-\eta(z) \frac{\partial}{\partial z}\right) P+\frac{\Phi}{\gamma H(0) \bar{\rho}(0)} \\
\frac{\partial P}{\partial t}=-\gamma g H(0) \bar{\rho}(0) \frac{\partial U}{\partial z}-g \bar{\rho}(0) \frac{\gamma-2}{2 \eta(z)} U \\
\frac{\partial \Phi}{\partial t}=-\frac{v(z)}{\eta(z)} g \bar{\rho}(0) U
\end{gathered}
$$


where $v(z)$ is positive:

$$
v(z)=\gamma-1+\gamma \frac{d H(z)}{d z}>0,
$$

that guarantee the positivity of energy density, defined at the Sec. 5, and the dimensionless height scale

$$
\eta(z)=\frac{H(z)}{H(0)}
$$

\subsection{Relation between pressure and entropy perturbations for acoustic and entropy modes}

The relation that links the pressure and entropy perturbation within the acoustic mode, for arbitrary stable stratification of 1D atmosphere can be obtained by substituting the Eq.(9) into the Eq.(8) [9]. As a result, the diagnostic relation between the pressure and entropy perturbations within the acoustic mode follows:

$$
P_{a}=\left(\frac{\gamma-2}{2 \eta(z)}+\gamma H(0) \frac{\partial}{\partial z}\right) \frac{\eta(z)}{v(z)} \Phi_{a}
$$

The first equation in the basic system (7) for $U_{0}=0$ fixes the diagnostic link in the stationary (entropy) mode:

$$
\Phi_{0}=\left(-\frac{\gamma-2}{2}+\gamma H(0) \eta(z) \frac{\partial}{\partial z}\right) P_{0}
$$

The relations (12) and (13) can be rewritten as

$$
\begin{aligned}
& P_{a}+D_{a} \Phi_{a}=0, \\
& \Phi_{0}+D_{0} P_{0}=0
\end{aligned}
$$

where the operators

$$
\begin{aligned}
& D_{a}=-\left(\frac{\gamma-2}{2 \eta(z)}+\gamma H(0) \frac{\partial}{\partial z}\right) \frac{\eta(z)}{v(z)}, \\
& D_{0}=-\left(-\frac{\gamma-2}{2}+\gamma H(0) \eta(z) \frac{\partial}{\partial z}\right)
\end{aligned}
$$

are the first order differential operators. We name the Eqs. $(14,15)$ as diagnostic relations, that define the acoustic and entropy mode in the 1D atmosphere with arbitrary stratification.

\subsection{Diagnostic equations}

Let us introduce operator-valued two-component vector:

$$
\left(\begin{array}{ll}
1 & D_{a}
\end{array}\right),
$$

and the column that represents the vector of state:

$$
\left(\begin{array}{l}
P \\
\Phi
\end{array}\right)
$$

where

$$
P=P_{a}+P_{0}, \Phi=\Phi_{a}+\Phi_{0}
$$

The action

$$
\left(\begin{array}{cc}
1 & D_{a}
\end{array}\right)\left(\begin{array}{c}
P \\
\Phi
\end{array}\right)=P+D_{a} \Phi=P_{a}+D_{a} \Phi_{a}+P_{0}+D_{a} \Phi_{0}=P_{0}+D_{a} \Phi_{0}=P_{0}-D_{a} D_{0} P_{0}
$$


defines the second order ordinary differential equation, we would name as the diagnostic one:

$$
\left(1-D_{a} D_{0}\right) P_{0}=P+D_{a} \Phi=f(z) .
$$

So, to extract the entropy mode, we need to solve the differential equation (22) with appropriate boundary conditions. Similar consideration with a solution form is presented at [11] in different units. The acoustic mode either could be extracted in the same manner or simply using the identity $P=P_{a}+P_{0}$.

It is pleasant to say, that there is a more simple alternative, leading to the differential diagnostic equation of the first order. The alternative option is realized by the row operator vector action on the column vector function

$$
\begin{gathered}
\left(\begin{array}{cc}
1 & D_{0}
\end{array}\right)\left(\begin{array}{c}
P \\
\Phi
\end{array}\right)=P+D_{0} \Phi=P_{a}+D_{0} \Phi_{a}+P_{0}+D_{0} \Phi_{0}= \\
=P_{a}+D_{0} \Phi_{a}=-D_{a} \Phi_{a}+D_{0} \Phi_{a}=\left(D_{0}-D_{a}\right) \Phi_{a} .
\end{gathered}
$$

The diagnostic relation (14) is taken into account. The derivation results in the first order equation

$$
\left(D_{0}-D_{a}\right) \Phi_{a}=P+D_{0} \Phi=g(z),
$$

see also [9], where the derivation is absent. The operator at the LHS of the second diagnostic equation (24) transforms as

$$
D_{0}-D_{a}=\frac{\gamma-2}{2}\left(\frac{1}{v(z)}+1\right)+\gamma\left(\frac{H(z)}{v(z)}\right)_{z}^{\prime}+\gamma H(z)\left(\frac{1}{v(z)}-1\right) \frac{\partial}{\partial z}
$$

or, by factorisation, it writes

$$
D_{0}-D_{a}=b(z)\left(\partial_{z}+a(z)\right)=b(z) \exp [-\psi] \partial_{z} \exp [\psi]=b(z)\left(\partial_{z}+\psi_{z}\right),
$$

where

$$
\begin{gathered}
b(z)=\gamma H(z)\left(\frac{1}{v(z)}-1\right), \\
a(z)=\frac{\frac{\gamma-2}{2}(v(z)+1)+\gamma \nu(z)\left(\frac{H(z)}{v(z)}\right)_{z}^{\prime}}{\gamma H(z)(1-v(z))}, \\
\psi=\int_{0}^{z} a(\zeta) d \zeta .
\end{gathered}
$$

\subsection{Solution of the first order diagnostic equation}

Taking the second alternative that looks more simple, we write the solution of the equation (24), that formally reads as

$$
\Phi_{a}=\left(D_{0}-D_{a}\right)^{-1}\left(P+D_{0} \Phi\right)=\exp [-\psi] \partial_{z}^{-1} \exp [\psi] b(z)^{-1} g(z) .
$$

It is expressed by direct integration formula

$$
\Phi_{a}=\exp [-\psi]\left(\int_{0}^{z} \exp [\psi] b(\zeta)^{-1} g(\zeta) d \zeta+C(t)\right)
$$

where $C(t)$ for a boundary regime propagation is defined from a condition at $z=0$ :

$$
\Phi_{a}(0)=\exp [\psi(0)] C(t) .
$$

It is convenient to choose $\psi(0)=0$, as in (29), hence

$$
C(t)=\Phi_{a}(0),
$$


Its choice would be in a consistency with the whole model, e.g. - either by results of measurements at the $z=0$ level or by results of the numerical calculations. Having oscillations, that is typical for waves, we fix the time $t^{\prime}$ for the choice $C\left(t^{\prime}\right)=0$.

\section{Applications to data of a numeric experiment}

3.1. On the dataset

We process the set of numerical experiment data consisting of horizontal coordinate, vertical coordinate, pressure, background pressure, density, background density, temperature, wave perturbation of temperature, wave perturbation of pressure, wave perturbation of density. The mentioned physical values are given as files such that for the fixed horizontal coordinate the vertical coordinate $z_{i}$ is presented for the range [0;500] $\mathrm{km}$ with the steps that varies with a height difference from $150 \mathrm{~m}$ to $2000 \mathrm{~m}$. The fixed time and horizontal coordinate seems to be convenient since the one-dimensional theory is considered. Data set was provided to authors by the sources, related to the paper [24]. The model is a numeric solution of the full two-dimensional nonlinear system of hydrothermodynamic balance equations. The program that processes the dataset allows solving the diagnostic equation (24) with reasonable accuracy of about a few percents, as estimated by the Runge rule.

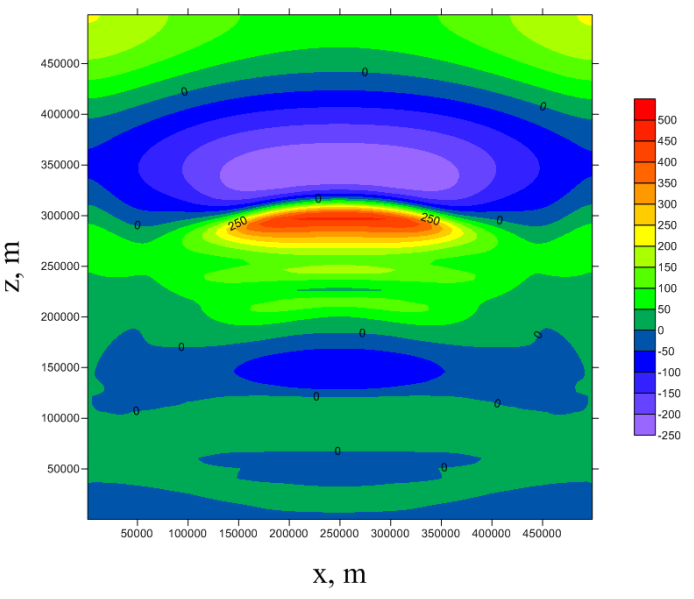

Figure 1. Wave perturbation of temperature from a local source of pressure fluctuations for the considered moment of time. Result of numeric modeling [24]. Courtesy of. Yu. Kurdjaeva.

According to the initial equation (24) and the diagnostic equations (30) and (31), such a study requires pressure and entropy data for constructing function $g(z)$ like:

$$
g(z)=P+D_{0} \Phi
$$

where the link of the variables $P, \Phi$ with original, pressure and density wave perturbations $p^{\prime}$ and $\rho^{\prime}$ directly measured ones, are described by the relations (4) and (5).

\subsection{Standard atmosphere $H(z)$ profile}

In this section we prepare the atmosphere characteristics for numerical calculations, starting from the atmospheric scale height calculated as

$$
H\left(z_{i}\right)=H_{i}=\frac{R_{s} T\left(z_{i}\right)}{g},
$$

where $z_{i}$ - $i$-row element of the discrete data array for vertical coordinate, $H_{i}$ - the value of the atmosphere scale height at the height $z_{i}, T\left(z_{i}\right)$ - the value of the temperature at the reference height $z_{i}, R_{s}=R / M=287.1 \mathrm{~J} /(\mathrm{kg} \cdot \mathrm{K})$ - the specific gas constant for dry air. 


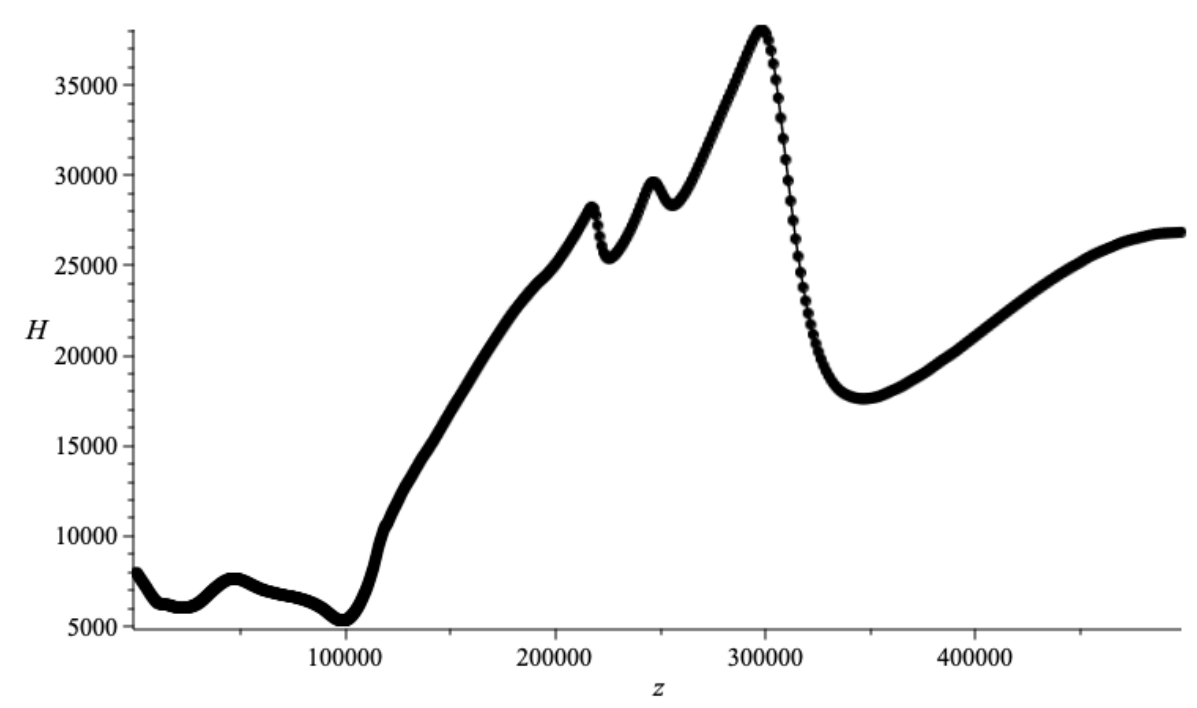

Figure 2. The height scale $H\left(z_{i}\right)$ profile obtained according to the formula (2).

\subsection{Discrete representation of functions and operators}

The functions (10) and (11) we approximate as follows. The expression shows how we estimate the derivatives (excluding the first and last points, where the left and right derivatives approximations are used),

$$
\begin{gathered}
v\left(z_{i}\right)=\gamma-1+\left.\gamma \frac{d H(z)}{d z}\right|_{z=z_{i}} \approx \gamma-1+\gamma \frac{H_{i+1}-H_{i-1}}{z_{i+1}-z_{i-1}}, \\
\eta\left(z_{i}\right)=\frac{H_{i}}{H(0)}
\end{gathered}
$$

where $H(0)$ - the atmosphere scale height at the lower boundary of the interval of $z$, which in the general case has the form $\left[z_{1} ; z_{2}\right]$. The ratio of (11) and (10) is approximated as

$$
\frac{\eta\left(z_{i}\right)}{v\left(z_{i}\right)}=\frac{\frac{H_{i}}{H(0)}}{\gamma-1+\gamma \frac{H_{i+1}-H_{i-1}}{z_{i+1}-z_{i-1}}}
$$

while its derivatives reads

$$
\left.\left(\frac{\eta(z)}{v(z)}\right)_{z}^{\prime}\right|_{z=z_{i}}=\frac{\frac{\eta\left(z_{i+1}\right)}{v\left(z_{i+1}\right)}-\frac{\eta\left(z_{i-1}\right)}{v\left(z_{i-1}\right)}}{z_{i+1}-z_{i-1}}
$$

From (24) yields:

$$
g_{i}\left(z_{i}\right)=P_{i}+D_{0, i} \Phi_{i}
$$

where according to the (17):

$$
D_{0, i}=-\left(\gamma H(0) \eta\left(z_{i}\right) \frac{\partial}{\partial z}-\frac{\gamma-2}{2}\right)=-\left(\gamma H_{i} \frac{\partial}{\partial z}-\frac{\gamma-2}{2}\right) .
$$

The functions $P$ and $\Phi$ are defined through a dataset using formulas (4), (5) that for the real $H\left(z_{i}\right)$ case are:

$$
P_{i}=P\left(z_{i}\right)=p^{\prime}\left(z_{i}\right) \cdot \exp \left(\int_{0}^{z_{i}} \frac{d z^{\prime}}{2 H\left(z^{\prime}\right)}\right) \approx p^{\prime}\left(z_{i}\right) \cdot \exp \left(\sum_{j=1}^{i} \frac{\Delta z_{j}}{2 H\left(z_{j}\right)}\right),
$$




$$
\Phi_{i}=\Phi\left(z_{i}\right)=\varphi^{\prime}\left(z_{i}\right) \cdot \exp \left(\int_{0}^{z_{i}} \frac{d z^{\prime}}{2 H\left(z^{\prime}\right)}\right) \approx \varphi^{\prime}\left(z_{i}\right) \cdot \exp \left(\sum_{j=1}^{i} \frac{\Delta z_{j}}{2 H\left(z_{j}\right)}\right) .
$$

So, finally the function $g\left(z_{i}\right)$ is approximated as:

$$
g\left(z_{i}\right)=P_{i}-\left.\gamma H_{i} \frac{\partial \Phi}{\partial z}\right|_{z=z_{i}}+\frac{\gamma-2}{2} \Phi_{i} \approx P_{i}-\gamma H_{i} \frac{\Phi_{i+1}-\Phi_{i-1}}{z_{i+1}-z_{i-1}}+\frac{\gamma-2}{2} \Phi_{i} .
$$

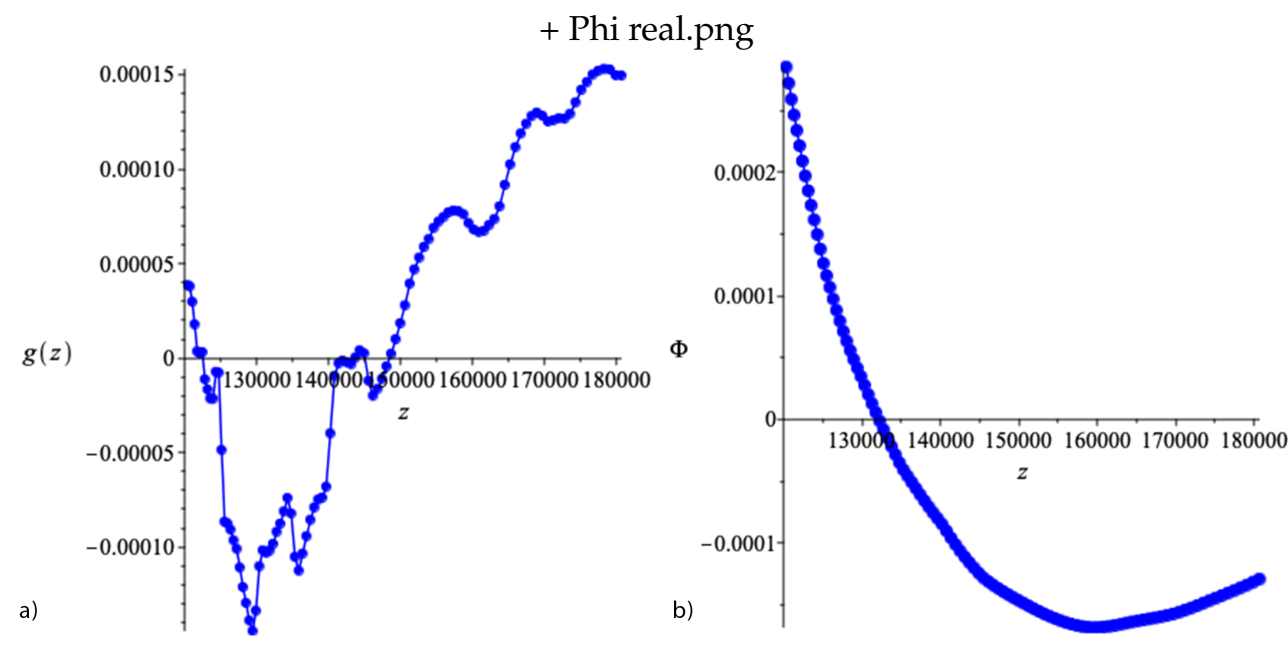

Figure 3. The plot a) is the graph of the function $g\left(z_{i}\right)$ obtained by the formula (44) and the plot b) is the graph of $\Phi\left(z_{i}\right)$ obtained by the formula (43) for the case of standard atmosphere $H\left(z_{i}\right)$ case.

\subsection{The wave entropy variable profile evaluation}

Let us reproduce (30) together with the functions (27)-(29)

$$
\begin{gathered}
b\left(z_{i}\right)=\gamma H(0) \eta\left(z_{i}\right)\left(\frac{1}{v\left(z_{i}\right)}-1\right)=\gamma H_{i}\left(\frac{1}{\gamma-1+\gamma \frac{H_{i+1}-H_{i-1}}{z_{i+1}-z_{i-1}}}-1\right), \\
a\left(z_{i}\right)=\frac{\frac{\gamma-2}{2}\left(\frac{1}{v\left(z_{i}\right)}+1\right)+\left.\gamma H(0)\left(\frac{\eta(z)}{v(z)}\right)_{z}^{\prime}\right|_{z=z_{i}},}{\gamma H(0) \eta\left(z_{i}\right)\left(\frac{1}{v\left(z_{i}\right)}-1\right)},
\end{gathered}
$$

and approximate the auxiliary function $\psi$ at the points $z_{i}$ as

$$
\psi_{i}=\int_{0}^{z_{i}} a(\zeta) d \zeta \approx \sum_{j=1}^{i} a\left(z_{j}\right) \Delta z_{j}
$$

Then the approximate solution of the second diagnostic equation (24) for the case of the standard atmosphere height scale values $H\left(z_{i}\right)$ at the points $z_{i}$ writes as:

$$
\Phi_{a}\left(z_{i}\right) \approx \exp \left(-\sum_{j=1}^{i} a\left(z_{j}\right) \Delta z_{j}\right) \sum_{j=1}^{i}\left[\frac{\exp \left(\sum_{j=1}^{i} a\left(z_{j}\right) \Delta z_{j}\right) g\left(z_{j}\right)}{b\left(z_{j}\right)}\right] \Delta z_{j} .
$$

Then the entropy mode contribution into the wave entropy variable can be found as:

$$
\Phi_{0}\left(z_{i}\right)=\Phi\left(z_{i}\right)-\Phi_{a}\left(z_{i}\right) .
$$




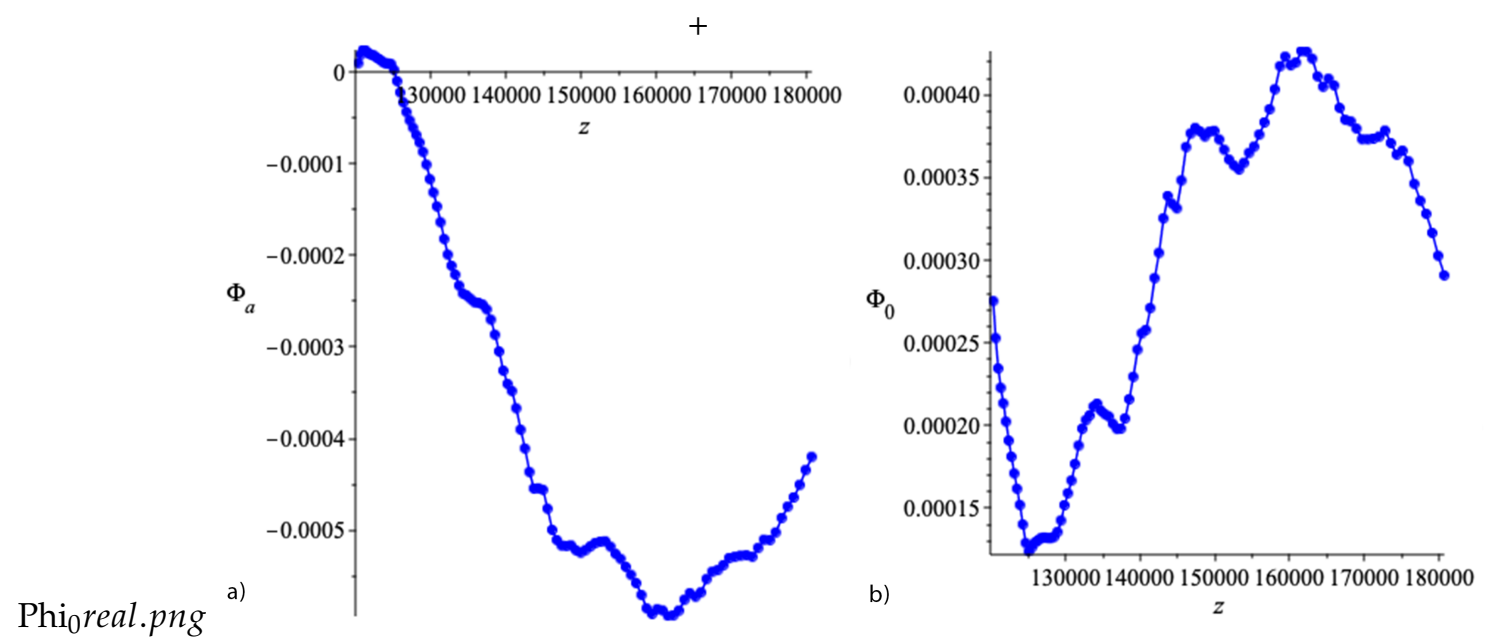

Figure 4. The left plot a) is the graph of the diagnostic equation solution $\Phi_{a}\left(z_{i}\right)$ obtained by the formula (48) and the right plot b) is the graph of $\Phi_{0}$ for the case of standard atmosphere height scale $H\left(z_{i}\right)$.

\section{An atmosphere model with approximate linear dependence of $H(z)$}

\subsection{The approximation parameters choice}

According to the graph for the atmosphere's scale height in Fig.2, we will focus on the approximately linear part in the interval $\mathrm{z} \in[120 ; 180] \mathrm{km}$.

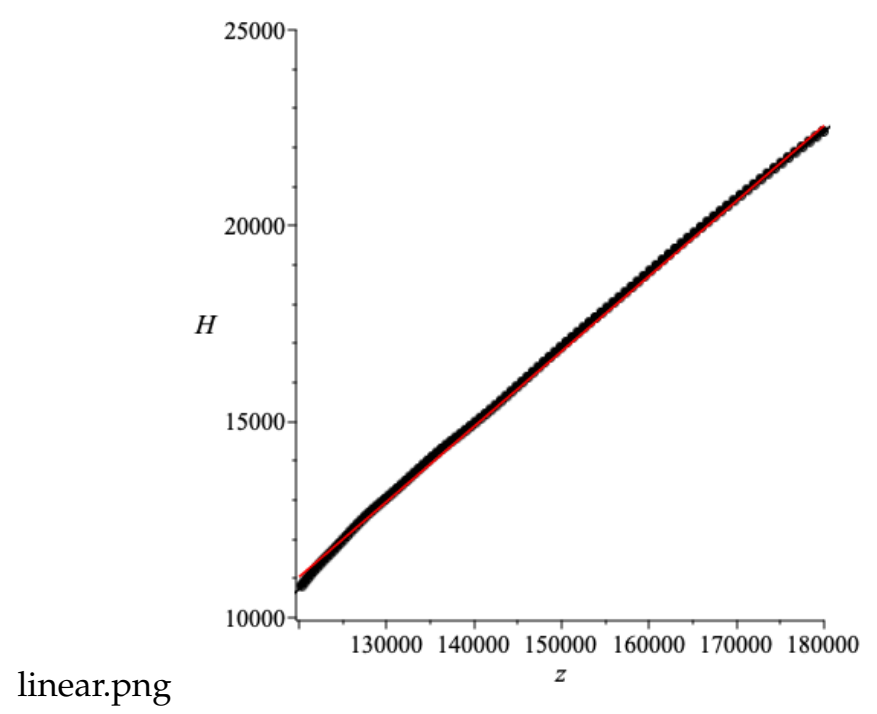

Figure 5. Standard atmosphere $H\left(z_{i}\right)$ for $z$ within $120 \mathrm{~km}$ to $180 \mathrm{~km}$ range (in black) and its linear approximation represented by formula (50) (in red)

To provide the model test, we put $H(z)$ depending linearly on the coordinate $z$ like:

$$
H(z)=\alpha z-H(0)
$$

where the curves at the Figure 5 adjustment is provided by the following choice

$$
\begin{gathered}
H(0)=H=12000 \mathrm{~m}, \\
\alpha=0.192 .
\end{gathered}
$$

The function $H(z)=0.192 z-12000$ graph is shown in red in Fig.5. 


\subsection{The linear $H(z)$ model and its basic forms of operators}

For the linear $H(z)$ case the functions (10) and (11) are:

$$
\begin{gathered}
v(z)=\gamma-1+\gamma \frac{d H(z)}{d z}=\gamma-1+\gamma \alpha=\gamma(\alpha+1)-1, \\
\eta(z)=\frac{H(z)}{H(0)}=\frac{\alpha z-H}{H}=\frac{\alpha}{H} z-1, \\
\frac{\eta(z)}{v(z)}=\frac{\alpha z / H-1}{\gamma(\alpha+1)-1}, \\
\frac{\partial}{\partial z} \frac{\eta(z)}{v(z)}=\frac{\alpha}{\gamma H(\alpha+1)-H^{\prime}},
\end{gathered}
$$

while the operator (17) takes the form:

$$
D_{0}=-\left(\gamma H(0) \eta(z) \frac{\partial}{\partial z}-\frac{\gamma-2}{2}\right)=-\left(\gamma(\alpha z-H) \frac{\partial}{\partial z}-\frac{\gamma-2}{2}\right) .
$$

Now for (24) with the relation (57) account we can evaluate the function $g(z)$ :

$$
g(z)=P+D_{0} \Phi=P-\left(\gamma(\alpha z-H) \frac{\partial}{\partial z}-\frac{\gamma-2}{2}\right) \Phi=P-\gamma(\alpha z-H) \frac{\partial \Phi}{\partial z}+\frac{\gamma-2}{2} \Phi
$$

where pressure and entropy perturbation functions (4)-(5) are:

$$
\begin{aligned}
& P=p^{\prime} \cdot \exp \left(\int_{z_{1}}^{z} \frac{d z^{\prime}}{2 H\left(z^{\prime}\right)}\right)=p^{\prime} \cdot \exp \left(\frac{1}{2 \alpha}\left(\ln (\alpha z-H)-\ln \left(\alpha z_{1}-H\right)\right)\right), \\
& \Phi=\varphi^{\prime} \cdot \exp \left(\int_{z_{1}}^{z} \frac{d z^{\prime}}{2 H\left(z^{\prime}\right)}\right)=\varphi^{\prime} \cdot \exp \left(\frac{1}{2 \alpha}\left(\ln (\alpha z-H)-\ln \left(\alpha z_{1}-H\right)\right)\right) .
\end{aligned}
$$

Here $z_{1}$ - initial coordinate or in the case of a discrete dataset for the vertical coordinate, that yields:

$$
\begin{aligned}
& P\left(z_{i}\right)=p^{\prime} \cdot \exp \left(\frac{1}{2 \alpha}\left(\ln \left(H\left(z_{i}\right)\right)-\ln \left(\alpha z_{1}-H\right)\right)\right) \\
& \Phi\left(z_{i}\right)=\varphi^{\prime} \cdot \exp \left(\frac{1}{2 \alpha}\left(\ln \left(H\left(z_{i}\right)\right)-\ln \left(\alpha z_{1}-H\right)\right)\right) .
\end{aligned}
$$

4.3. The wave variable entropy profile evaluation for the linear $H(z)$

The functions (27) and (28) for the case of a linear dependence $H(z)$ are evaluated in explicit form:

$$
\begin{gathered}
b(z)=\gamma(\alpha z-H)\left(\frac{1}{\gamma(\alpha+1)-1}-1\right)=\gamma(\alpha z-H) \frac{2-\gamma(\alpha+1)}{\gamma(\alpha+1)-1}, \\
a(z)=\frac{\frac{\gamma-2}{2}\left(\frac{1}{\gamma(\alpha+1)-1}+1\right)+\gamma H \frac{\alpha}{\gamma H(\alpha+1)-H}}{\gamma(\alpha z-H)\left(\frac{1}{\gamma(\alpha+1)-1}-1\right)}=\frac{-1}{2(\alpha z-H)},
\end{gathered}
$$




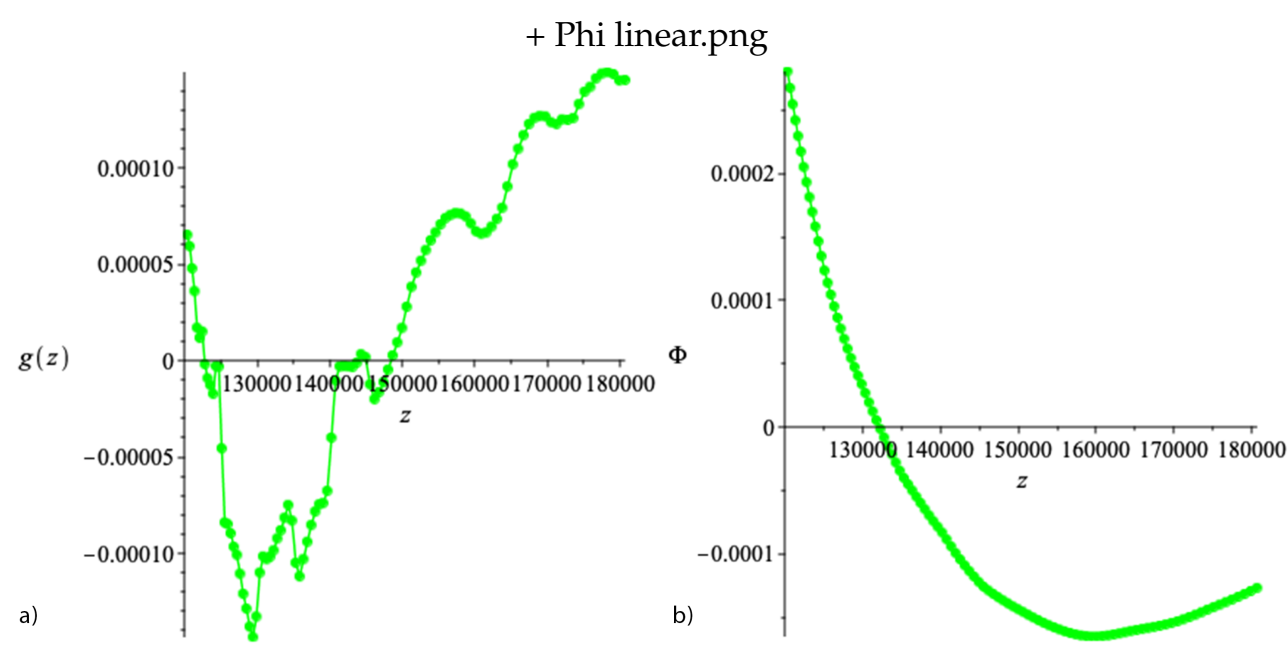

Figure 6. The left figure a) is the graph of the function $g\left(z_{i}\right)$ obtained by the formula (58) and the right figure b) is the graph of $\Phi\left(z_{i}\right)$ obtained by the formula (62) for the case of linear dependence on the height scale $H(z)$.

The auxiliary function (29) is defined within the limits of integration, that is changed compared with (29):

$$
\psi=\int_{z_{1}}^{z} a(\zeta) d \zeta=-\frac{1}{2} \int_{z_{1}}^{z} \frac{d \zeta}{(\alpha \zeta-H)}=-\frac{1}{2 \alpha}\left(\ln (\alpha z-H)-\ln \left(\alpha z_{1}-H\right)\right) .
$$

Solution (31) for the linear $H(z)$ takes the form:

$$
\Phi_{a}=\frac{\gamma(\alpha+1)-1}{\gamma(2-\gamma(\alpha+1))} \exp \left[\frac{\ln (\alpha z-H)}{2 \alpha}\right] \int_{z_{1}}^{z} \exp \left[-\frac{\ln (\alpha \zeta-H)}{2 \alpha}\right] \frac{g(\zeta)}{(\alpha \zeta-H)} d \zeta
$$

or, by the properties of exponential and logarithmic functions, it is rewritten as

$$
\Phi_{a}=\frac{\gamma(\alpha+1)-1}{\gamma(2-\gamma(\alpha+1))}(H(z))^{\frac{1}{2 \alpha}} \int_{z_{1}}^{z} g(\zeta)(H(\zeta))^{-\frac{1}{2 \alpha}-1} d \zeta
$$

Phi ${ }_{0}$ linear.png
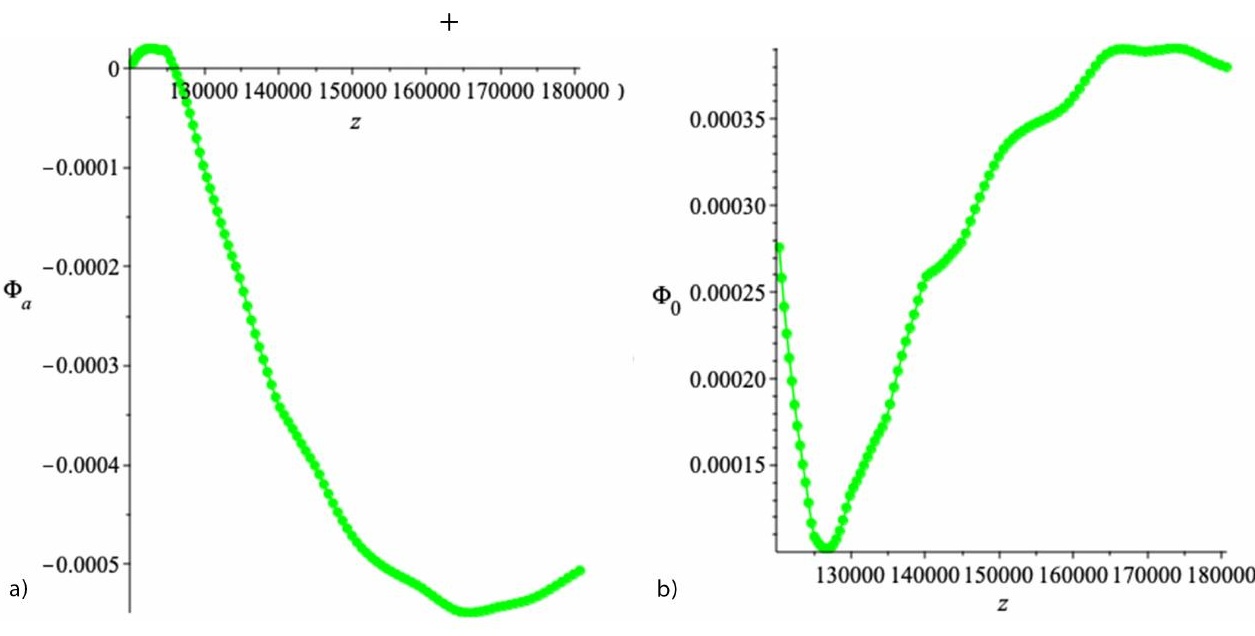

Figure 7. a) is the graph of the solution $\Phi_{a}(z)$ obtained by the formula (62) and b) is the graph of $\Phi_{0}(z)$ for the case of linear dependence $H(z)$. 


\section{Comparison of the models and discussion of the results}
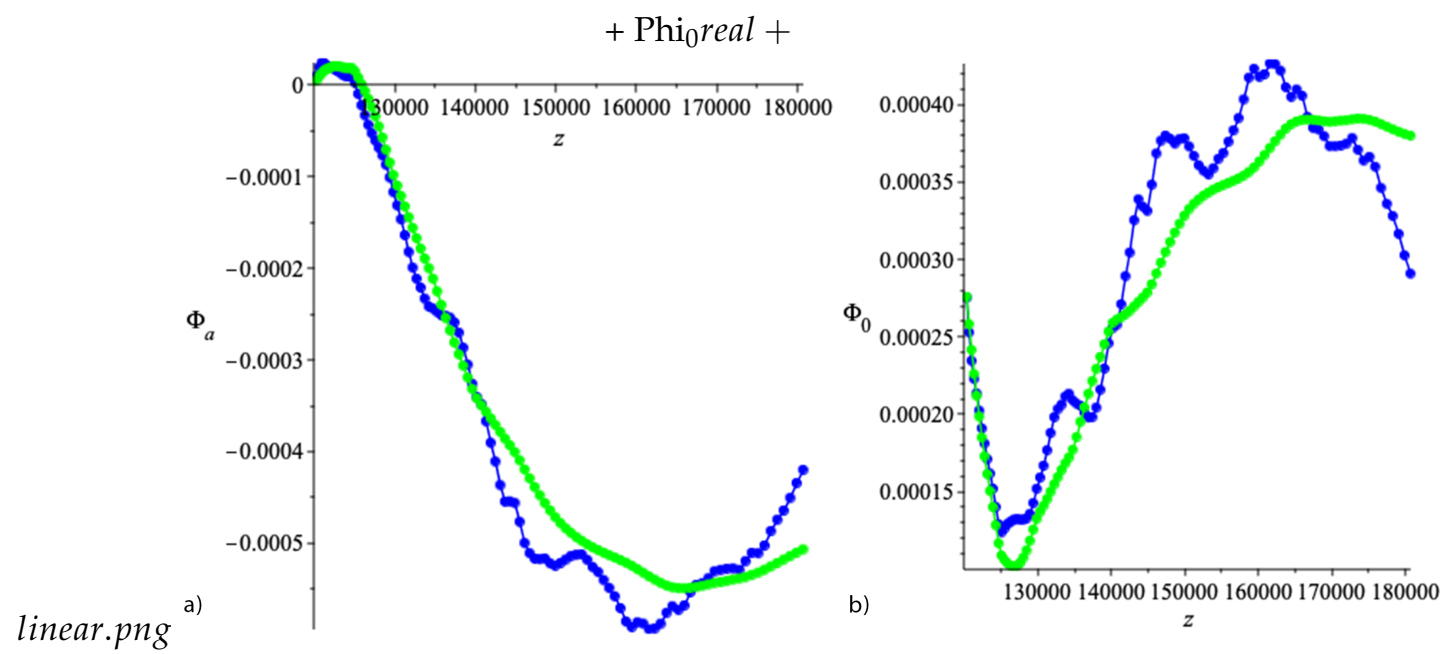

Figure 8. Comparison of the acoustic mode contribution $\Phi_{a}$ (a) and the entropy mode one $\Phi_{0}$ (b) solutions of the diagnostic equation for the cases of standard atmosphere (blue) and linear height scale dependence $H(z)$ model (50) (green).

The authors believe that the analytical models are more desirable than numerical methods, which are usually time-consuming, require a high-performance computer, and special attention to underlying algorithms, their convergence, and stability investigation. On the other hand, reasonably simple analytical models also, when complemented by a numerical approach, are much more efficient.

Speaking about the modes extraction at the level of the pressure-entropy vector disturbances field we observe the difference of the results, visible at the plots of the Figure 8. The difference (by module about 5 percents) originated from the significant non-coincidence of the functional parameter $v(z)$. Namely, it is constant, in the case of the model (approximately equal to 0.79 ), but vary, oscillating from 0.73 to 0.92 , being calculated directly from standard atmosphere data $H(z)$, differentiating in (10) via the conventional derivative approximation. Estimation of the energy $E(z)$, the total energy of all modes at the coordinate range $[0, z]$, is given by the following expression

$$
E(z)=\frac{1}{2} \int_{0}^{z} d z\left(\bar{\rho} V^{2}+\frac{p^{\prime 2}}{\gamma \bar{p}}+\frac{\phi^{\prime 2}}{\gamma v(z) \bar{p}}\right)
$$

see the profiles at the Figure 9.

Note, that the energy profiles for the cases of the direct standard atmosphere use and the model, based on explicit linear dependence application gives the curves which difference is scarcely visible (the difference about the percent), hence we propose to use the total energy values and the profiles (68) for the model mode weights estimation.

\section{Conclusions}

The main result of the presented work constitutes in the diagnostic equation, which solution gives the vertical profile of the acoustic mode contribution in the entropy perturbation. This result is illustrated by application to realistic numerical modeling of the atmosphere perturbation by a source positioned near Earth surface. The next result of the study presents the model of the diagnostic algorithm that uses the restricted heights interval, at which the $\mathrm{H}(\mathrm{z})$ dependence is very close to linear. Its restriction guarantees the stability condition and the energy density positively defined. The dependence of explicit approximation on $z$ allows proceeding with the diagnostic equation solution in explicit form. The resulting diagnostic operations are compared with ones of numerical calculations at the whole available heights range under consideration. The extracted acoustical and 


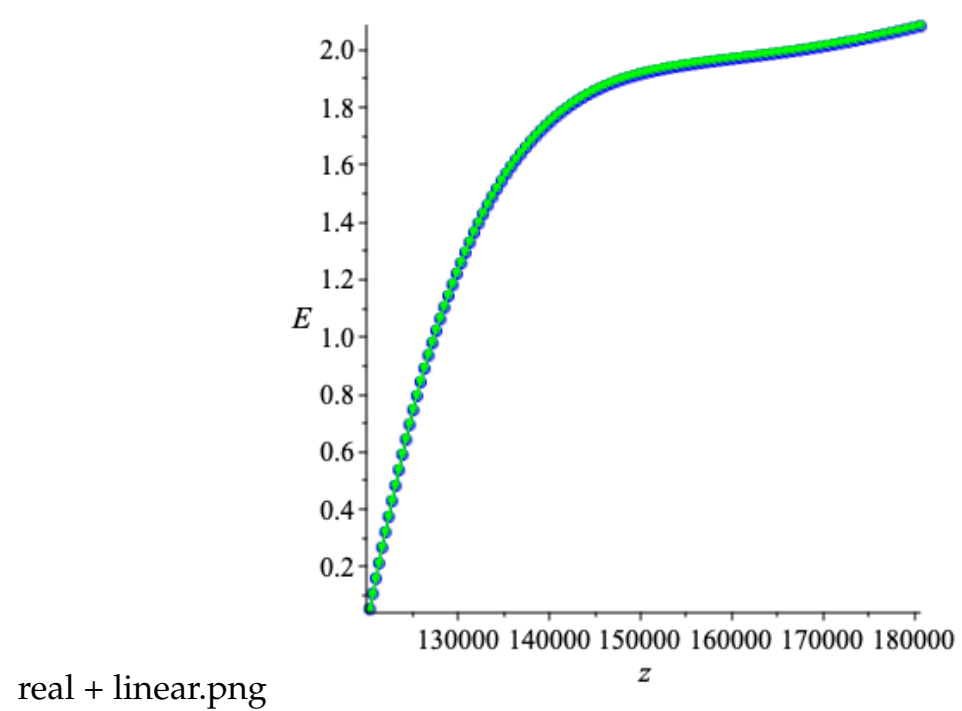

Figure 9. Energy calculated by the equation (68) for the cases of standard atmosphere (blue) and linear dependence (green) of the height scal $H(z)$ for $z \in[120 ; 180] \mathrm{km}$., see the relation (50)

entropy modes contributions in perturbation of the gas entropy profiles are plotted and compared with the models'.

One of the important ingredients of diagnostics is the possibility to estimate the relative weight of a mode contribution. It is also important to evaluate an error of this estimation, cumulative measurements errors and the theoretical and numerical discrepancies. Such possibility is directly based on the energy density definition with the positive functional parameter $n u$ at a height range under consideration. It, as known, leads to the norm definition in a functional space of the state vector.

Author Contributions: Conceptualization, S.L.,methodology, S.L.; software, E.S.; validation, S.L., E.S.; formal analysis, S.L., E.S.; investigation, S.L.; resources, S.L., E.S.; data curation, E.S.; writingoriginal draft preparation, S.L.; writing—review and editing, S.L., E.S.; visualization, E.S.; supervision, S.L.; project administration, S.L.; All authors have read and agreed to the published version of the manuscript.

Acknowledgments: The authors are thankful to Ivan Karpov for important advice and Julia Kurdyaeva for the share of numerical simulation data.

Conflicts of Interest: The authors declare no conflict of interest.

\section{References}

1. Brekhovskikh, L.M.; Godin A.O. Acoustics of layered media; Springer-Verlag, Berlin, 1990.

2. Pedloski, J. Geophysical fluid dynamics; Springer-Verlag, New York, 1987.

3. Gordin, A.V. Mathematical problems of hydrodynamical weather prediction. Analytical aspects; Gydrometeoizdat [in Russian], Leningrad, 1987.

4. Borovikov, V.A.; Kelbert, M.Ya. Adaptation of initial conditions for internal waves in weakly compressible liquid; Nauka [in Russian], Tbilisi, 1985.

5. Leble, S.B. Nonlinear waves in waveguides with stratification; Springer-Verlag, Berlin, 1990.

6. Leble, S.; Vereshchagina, I. Problem of disturbance identification by measurement in the vicinity of a point, TASK QUARTERLY 2016, 20(2) 131-141.

7. Karpov, I.V.; Kshevetsky, S.P.; Borchevkina O.P.; Radievsky A.V.; Karpov A.I. Disturbances of the upper atmosphere and ionosphere caused by acoustic-gravity wave sources in the lower atmosphere, Russian Journal of Physical Chemistry B 2016, 10(1), 127-132.

8. Leble, S.; Perelomova A. Problem of proper decomposition and initialization of acoustic and entropy modes in a gas affected by the mass force, Applied Mathematical Modelling 2013, 37, 629-635.

9. Leble, S.; Perelomova, A. Decomposition of acoustic and entropy modes in a non-isothermal gas affected by a mass force, Archives of Acoustics 2018, 43(3), 497-503.

10. Leble, S.; Perelomova, A. Dynamical projectors method in hydro- and electrodynamics; CRC Press, Taylor and Frensis group, 2018. 
11. Brezhnev, Yu.; Kshevetsky, S.; Leble, S. Linear initialization of hydrodynamical fields, Atmospheric and Oceanic Physics 1994, 30(10), 84-88.

12. Sun, L.; Robinson, W. A.; Chen, G. The predictability of stratospheric warming events: more from the troposphere or the stratosphere?; Journal of the Atmospheric Sciences 2012, 69(2), 768-783.

13. Butler, A. H.; Sjoberg, J. P.; Seidel, D. J.; Rosenlof, K. H. A sudden stratospheric warming compendium, Earth System Science Data 2017, $9(1), 63-76$.

14. Leble, S.; Smirnova, E. Tsunami-Launched Acoustic Wave in the Layered Atmosphere: Explicit Formulas Including Electron Density Disturbances, Atmosphere 2019, 10, 629.

15. Belikovich, V.V.; Benediktov, E.A.; Tolmacheva, A.V.; Bakhmet'eva, N.V. Ionospheric Research by Means of Artificial Periodic Irregularities; Copernicus GmbH: Katlenburg-Lindau, Germany, 2002.

16. Bakhmetieva, N.V.; Grigor'ev, G.I.; Tolmacheva, A.V. Artificial periodic irregularities, hydrodynamic instabilities, and dynamic processes in the mesosphere-lower thermosphere, Radiophys. Quantum Electron 2011, 53, 623-637.

17. Leble, S.; Vereshchagin, S.; Bakhmetieva, N.; Grigoriev, G. On the Diagnosis of Unidirectional Acoustic Waves as Applied to the Measurement of Atmospheric Parameters by the API Method in the SURA Experiment, Atmosphere 2020, 11, 924.

18. Leble, S.; Vereshchagin, S.; Vereshchagina, I. Algorithm for the Diagnostics of Waves and Entropy Mode in the Exponentially Stratified Atmosphere, Russian Journal of Physical Chemistry B 2020, 14 (2), 371-376.

19. Perelomova, A. Weakly nonlinear dynamics of short acoustic waves in exponentially stratified gas, Archives of Acoustics 2009,34 (2), 127-143.

20. Perelomova, A. Nonlinear dynamics of vertically propagating acoustic waves in a stratified atmosphere, Acta Acustica 1998, 84 , 1002-1006.

21. Perelomova, A. Nonlinear dynamics of directed acoustic waves in stratified and homogeneous liquids and gases with arbitrary equation of state, Archives of Acoustics 2000, 25 (4), 451-463.

22. U.S. Standard Atmosphere, U.S. Government Printing Office, Washington, D.C., 1976.

23. Kshevetskii, S.P.; Kurdyaeva, Y.A.; Gavrilov, N.M.; Karpov, I.V. Simulation of Vertical Propagation of Acoustic-Gravity Waves in the Atmosphere based on Variations of Atmospheric Pressure and Research of Heating of the Upper Atmosphere by Dissipated Waves, Proceedings of V International conference Atmosphere, Ionosphere, Safety 2016, 468-473.

24. Kshevetskii, S.P.; Kurdyaeva, Yu.A. The Numerical Study of Impact Of Acoustic-Gravity Waves from the Pressure Source on The Earth's Surface on the Thermosphere Temperature, Trudy' Kol'skogo nauchnogo czentra RAS 2016, 4(38), 161-166.

25. Kurdyaeva, Y.A.; Kshevetski, S.P.; Gavrilov, N.; Kulichkov, S.N. Correct boundary conditions for the high-resolution model of nonlinear acoustic-gravity waves forced by atmospheric pressure variations, Pure and Applied Geophysics 2018, 175, 3639-3652. 